\title{
A Survey on the Modeling of Magnetic Tunnel Junctions for Circuit Simulation
}

\author{
Hyein Lim, Seungjun Lee, and Hyungsoon Shin \\ Department of Electronics Engineering, Ewha Womans University, 11-1 Daehyun-Dong, Seodaemoon-Gu, \\ Seoul 120-750, Republic of Korea \\ Correspondence should be addressed to Seungjun Lee; slee@ewha.ac.kr and Hyungsoon Shin; hsshin@ewha.ac.kr
}

Received 5 February 2016; Accepted 6 April 2016

Academic Editor: Hongyang Zhao

Copyright (C) 2016 Hyein Lim et al. This is an open access article distributed under the Creative Commons Attribution License, which permits unrestricted use, distribution, and reproduction in any medium, provided the original work is properly cited.

\begin{abstract}
Spin-transfer torque-based magnetoresistive random access memory (STT-MRAM) is a promising candidate for universal memory that may replace traditional memory forms. It is expected to provide high-speed operation, scalability, low-power dissipation, and high endurance. MRAM switching technology has evolved from the field-induced magnetic switching (FIMS) technique to the spin-transfer torque (STT) switching technique. Additionally, material technology that induces perpendicular magnetic anisotropy (PMA) facilitates low-power operation through the reduction of the switching current density. In this paper, the modeling of magnetic tunnel junctions (MTJs) is reviewed. Modeling methods and models of MTJ characteristics are classified into two groups, macromodels and behavioral models, and the most important characteristics of MTJs, the voltage-dependent MTJ resistance and the switching behavior, are compared. To represent the voltage dependency of MTJ resistance, some models are based on physical mechanisms, such as Landau-Lifshitz-Gilbert (LLG) equation or voltage-dependent conductance. Some behavioral models are constructed by adding fitting parameters or introducing new physical parameters to represent the complex switching behavior of an MTJ over a wide range of input current conditions. Other models that are not based on physical mechanisms are implemented by simply fitting to experimental data.
\end{abstract}

\section{Introduction}

Spin-transfer torque-based magnetoresistive random access memory (STT-MRAM) has emerged as a promising candidate for the next generation of nonvolatile memory. Traditional forms of memory, such as DRAM or flash memory, have reached their limits in terms of scaling, power consumption, endurance, and other important parameters. STT-MRAM has many advantages, including nonvolatility, high speed, scalability, low-power dissipation, and high endurance $[1,2]$.

To facilitate the commercialization of STT-MRAM, it is important to satisfy the requirements of scalability and low-power dissipation. Progress in switching technology and material developments have enabled reductions in the switching current density according to the demands of lowpower consumption and high-speed operation. Advances in switching technology from field-induced magnetic switching (FIMS) to STT switching and material technology from inplane devices to perpendicular magnetic anisotropy (PMA) devices have accelerated the development of STT-MRAM.
An accurate and efficient model is required to simulate and design an MTJ-based STT-MRAM. The model should provide an exact physical description to precisely represent the characteristics of the MTJ. Also, the model must be compatible with circuit-level simulators, such as SPICE. There are many MTJ models, including macromodels, micromagnetic models, and behavioral models. Macromodels are composed of circuit elements, such as resistors, capacitors, and voltage sources. They are beneficial for their compatibility with circuit-level simulators, but the number of circuit elements increases with the complexity of the MTJ's dynamic characteristics. Micromagnetic models have an advantage in terms of accuracy because they model the movement of individual magnetization. Micromagnetic models are usually based on the LLG equation and useful for understanding the physical switching process of a single MTJ cell. Their importance has increased as the technology node has decreased such that the movement of individual magnetization has become important. However, due to the complexity of these models, the micromagnetic model is rather inappropriate for 


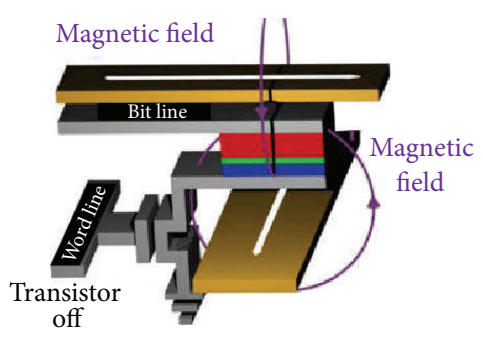

(a)

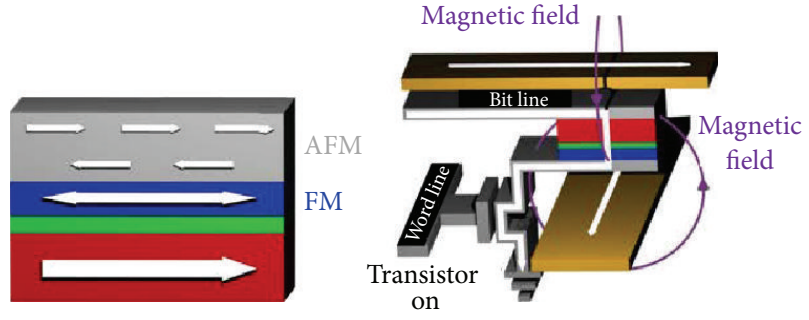

(b)

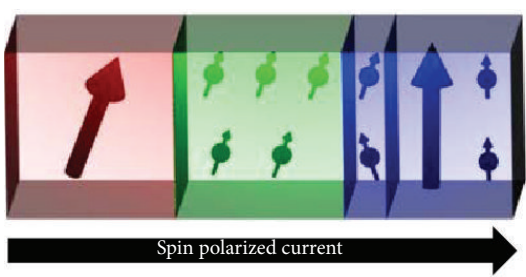

(c)

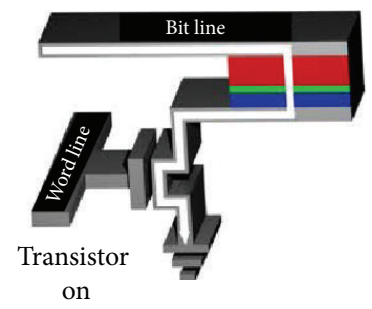

on

FIGURE 1: Development trend in MRAM switching technology. (a) FIMS, (b) TAS, and (c) STT. Reprinted from [5].

the simulation of large arrays of STT-MRAM. Behavioral models are often written in a hardware description language, such as Verilog-A or Verilog-C language, which are compatible with circuit-level simulators. Behavioral models are beneficial for circuit simulation because the model represents the characteristics of an MTJ analytically, requiring only one element, whereas a macromodel requires many circuit elements.

Some studies have compared the behavioral models and LLG equation-based micromagnetic model $[3,4]$. In [3], the LLG approach exhibits a faster simulation speed than the behavioral approach. However, [4] reports that the model based on the LLG equation requires a longer simulation time than the behavioral model. Therefore, the conclusions regarding these modeling methods are still controversial.

The remainder of this paper is organized as follows. The switching technology and material technology that underlie MRAM are reviewed in the next chapter. In the following chapters, models of MTJ characteristics are organized into two categories, macromodels and behavioral models, and the most important characteristics of MTJs, voltage-dependent MTJ resistance and switching behavior, are compared.

\section{MRAM Technology}

2.1. Development History of Switching Technology. Switching technology evolved from the FIMS technique to the STT switching technique over the course of many years, as shown in Figure 1 [5].

In the early days, MRAM used the FIMS switching technique [19], which switches the magnetization using a magnetic field induced by the conducting lines adjacent to the MTJ, as shown in Figure 1(a). If the current flow is sufficient to produce a critical magnetic field, the direction of magnetization in the ferromagnetic layer switches to the opposite direction. However, the FIMS technique has several disadvantages and limits. The FIMS technique occupies a large area $\left(20-30 \mathrm{~F}^{2}\right)$ for one cell, and, thus, it is not possible to achieve a high density. The current required increases with decreases in cell size because the coercivity field that resists the switching becomes larger. In addition, write errors can occur due to interference induced by the magnetic field from adjacent cells as the distance between cells becomes smaller.

Another switching technique is the thermally assisted switching (TAS) technique [20]. The TAS technique has received considerable attention as a means of overcoming the limitations of the FIMS technique, namely, write selectivity and power consumption. This technique depends on a combination of a magnetic field and heat, as shown in Figure 1(b). Read operations are conducted at room temperature, whereas write operations are conducted at an elevated temperature by injecting current directly into the MTJ. The high temperature makes the magnetization of the ferromagnetic layer easily switches using a weak magnetic field, and, thus, the power consumption is reduced. In addition, the current flows directly through the device that is selected to be written to resolve the write selectivity issue. However, the scaling and stability issues still remain.

The STT switching technique was proposed to overcome the limitations of switching techniques presented above. This technique switches the magnetization by injecting current directly into the MTJ [21] without using any external magnetic field, as shown in Figure 1(c). When current flows into the ferromagnetic layer, the current is polarized, and the polarized current induces STT to the magnetization of the ferromagnetic layer to conserve the spin angular momentum. If the magnitude of current exceeds a given threshold, the magnetization is switched by STT. This technique has the advantages of scalability and high density because there is no need for external conducting lines to induce the magnetic field. Therefore, the STT-MRAM cell area can be reduced to approximately $6 \mathrm{~F}^{2}$, which is considerably smaller than the areas needed for the FIMS or TAS techniques. Additionally, the magnitude of the switching current can be decreased, which reduces the power consumption. 
The next promising switching technique after STT switching is the voltage-induced switching technique. The current density of STT switching is approximately $\sim \mathrm{MA} / \mathrm{cm}^{2}$, and it is difficult to reduce this current density. Therefore, the STT switching technique still requires a large CMOS transistor, which is inefficient for power consumption. The voltage-induced switching technique is expected to reduce the switching current density to $<10^{5} \mathrm{~A} / \mathrm{cm}^{2}$. The principle of the voltage-induced switching technique exploits voltagecontrolled magnetic anisotropy. The energy barrier between the ferromagnetic layer and oxide layer is reduced during the switching process by applying voltage pulses to MTJ. However, this technique requires the magnetic field to determine the switching direction.

2.2. Material Technology of STT-MRAM. Low-power operation is one of the most important requirements for accelerating the commercialization of STT-MRAM. The switching current density of STT-MRAM must be decreased to achieve low-power consumption and high-speed operation. Various methods to reduce the switching current density of MTJ have been proposed such as decreasing the free layer thickness [22] or using a double-oxide tunnel barrier layer [23]. Lowering the switching current density is likely to cause thermal instability in in-plane magnetic anisotropy (IMA) devices because the anisotropy field decreases with decreasing cell size. There are many studies on PMA devices [24-26] that offer a lower switching current density than IMA devices without the problem of thermal instability in IMA devices. PMA devices have been reported to have the advantages of scalability, thermal stability, low current density, high speed, and low-power consumption.

Several methods have been proposed to obtain the characteristics of PMA by using such materials as rare-earth/transition metal alloy electrodes [27, 28]. Recently, orthogonal spin-transfer MRAM (OST-MRAM) and interfacial-induced PMA have received considerable attention. OST-MRAM employs a second spin-polarizing layer magnetized perpendicularly to the in-plane free layer $[6,29]$. The conventional IMA device has a small STT at the initial stages of the switching process, and, thus, it is difficult to reduce the switching time to the subnanosecond scale. The initial STT is increased by adding second perpendicular polarizer which accelerates the switching time to the picosecond scale by eliminating the initial incubation time. Interfacial-induced PMA uses and Fe-rich $\mathrm{CoFeB}$ free layer $[25,30]$ or a Ta seed layer instead of the $\mathrm{Ru}$ seed layer [7]. These studies have presented excellent experimental results in terms of low switching current density and high switching speed. Switching current density is one of the most important parameters of STT-MRAM due to its effect on switching speed and thermal stability factor.

The critical switching current density of an IMA device at zero temperature is given by $[31,32]$

$$
\begin{aligned}
J_{\mathrm{C} 0} & =\frac{\alpha \gamma e\left(\mu_{0} M_{S}\right) t_{\text {free }}}{\mu_{\mathrm{B}} g}\left(H_{A}+\frac{H_{d}}{2}\right), \\
g & =\frac{P}{2\left(1+P^{2} \cos \theta\right)},
\end{aligned}
$$

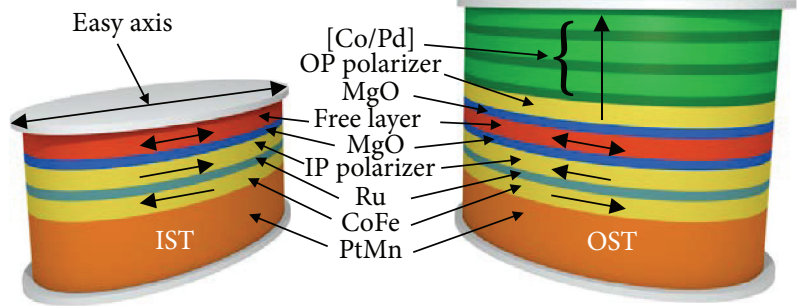

(a)

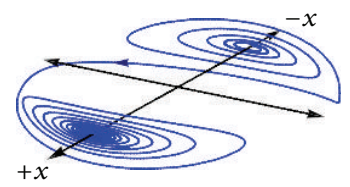

(c) $0.85 \mathrm{~ns}$ pulse (b)

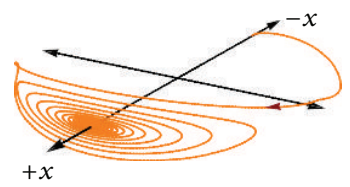

(d) 0.12 ns pulse
Figure 2: (a) Schematic of an IMA device and (b) a PMA device made by adding a perpendicular polarizer to an IMA device. ((c) and (d)) Simulated macrospin trajectories of magnetizations in (c) IMA device and (d) PMA device. Reprinted from [6].

where $\alpha$ is the Gilbert damping coefficient, $\gamma$ is the gyromagnetic ratio, $e$ is the electron charge, $\mu_{0}$ is the magnetic permeability, $M_{s}$ is the saturation magnetization, $t_{\text {free }}$ is the thickness of the free layer, $H_{A}$ is the in-plane anisotropy field, $H_{d}$ is the demagnetization field, $\mu_{\mathrm{B}}$ is the Bohr magneton, $g$ is the spin-transfer efficiency, $P$ is the electron polarization percentage, and $\theta$ is the angle between the magnetizations of the free layer and reference layer. As described in (1a), an IMA device makes a trade-off between the critical switching current density and thermal stability factor. The thermal stability factor is expressed as $E / k_{\mathrm{B}} T$, where $E=\mu_{0} M_{s} H_{K} V / 2$ is the energy barrier, $H_{K}$ is the anisotropy field, $V$ is the volume of the free layer, $k_{\mathrm{B}}$ is the Boltzmann constant, and $T$ is temperature. These relationships illustrate that thermal instability can be induced by reducing the switching current density.

PMA reduces the switching current density by simultaneously canceling the demagnetization field and conserving the energy barrier $E$ [33]. The demagnetization field is decreased by adding a perpendicular polarizer to the IMA device $[6,29]$, as shown in Figure 2. The critical switching current density is described as

$$
J_{\mathrm{C} 0}=\frac{\alpha \gamma e\left(\mu_{0} M_{S}\right) t_{\text {free }}}{\mu_{\mathrm{B}} g}\left(H_{A}+\frac{\left(H_{d}-H_{k \perp}\right)}{2}\right),
$$

where $H_{k \perp}$ is the perpendicular magnetic anisotropy field [34]. Rowlands et al. [6] showed that adding a perpendicular polarizer to an IMA device can significantly increase the switching speed to as high as $0.1 \mathrm{~ns}$ and decrease the write energy to as low as $0.4 \mathrm{pJ}$ (Figure 3). By adding a perpendicular polarizer, the perpendicular magnetic anisotropy field reduces the demagnetization field, and spin torque from the perpendicular polarizer pushes the magnetization of the free layer to the out-of-plane direction so that faster switching can occur than in conventional IMA devices. 


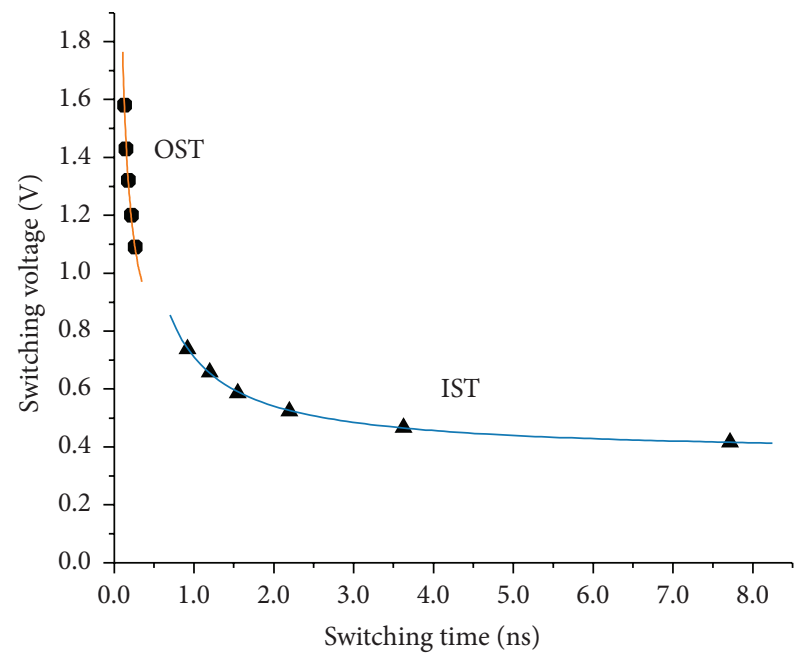

(a)

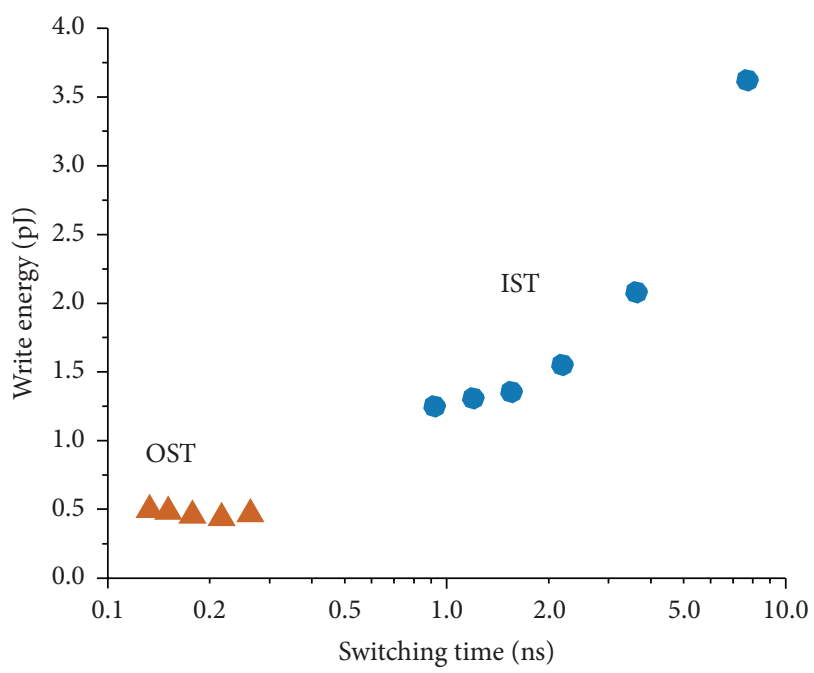

(b)

FIGURE 3: Comparison between an IMA device and PMA device. (a) Switching voltage as a function of pulse width at 50\% switching probability. (b) Energy per write at 50\% switching probability as a function of switching time. Reprinted from [6].

The other method of decreasing switching current density is to make a fully perpendicular ferromagnetic layer such that the perpendicular anisotropy field becomes sufficiently large to overcome the demagnetization field $[7,30,35,36]$. In this case, the demagnetization field is fully cancelled, and, thus, the critical switching current density is decreased, as is shown by [34]

$$
J_{\mathrm{C} 0}=\frac{\alpha \gamma e\left(\mu_{0} M_{\mathrm{S}}\right) t_{\text {free }}}{2 \mu_{\mathrm{B}} g} H_{k \perp}
$$

It is noted that the critical switching current density is proportional to the perpendicular magnetic anisotropy field. Furthermore, a fully PMA device has an advantage of scalability because the thermal stability is maintained while minimizing the energy barrier. Worledge et al. reported [7] that the switching speed of a PMA device is 8 times faster and the critical switching voltage is lower than those of an IMA device, as shown in Figure 4.

According to the ITRS roadmap 2013 [37], STT-MRAM material technology will be developed to use PMA in 2017 as the technology node decreases as shown in Table 1. Therefore, a thorough investigation of PMA devices must be conducted.

\section{Modeling of MTJ Characteristics}

One of the major obstacles for the commercialization of STT-MRAM is the absence of an accurate and efficient circuit model for MTJs. A model that is physically accurate and efficient for a circuit-level simulation would be useful for the design optimization of large-scale STT-MRAMs. If the model accurately represents the characteristics of an MTJ, overestimation of design margins can be avoided to decrease the cell area and power consumption. Additionally, a computationally efficient circuit model is essential for

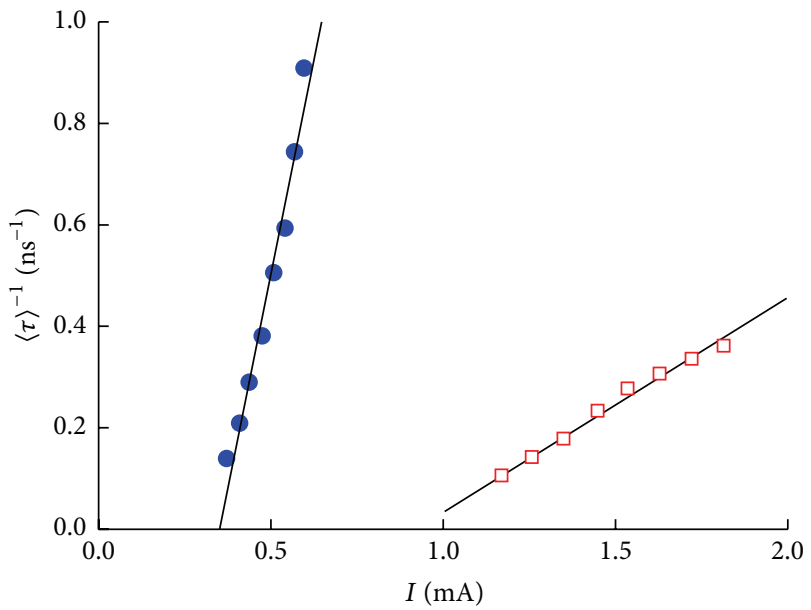

FIGURE 4: Switching speed as a function of current for a PMA device (blue circles) and an IMA device (red squares). Reprinted from [7].

reducing simulation time so that the design cycle of STTMRAM could be accelerated.

In this chapter, modeling methods and a model of MTJ characteristics will be reviewed. Models for MTJ resistance with voltage dependence and switching behavior modeling, which are two of the most important characteristics of MTJs, are surveyed. For the two-characteristic modeling, models for circuit-level simulation can be classified into two groups, macromodels (or structural models) and behavioral models.

3.1. Voltage-Dependent MTJ Resistance Modeling. Many experiments have demonstrated that the MTJ resistance has a voltage dependence $[38,39]$. The MTJ resistance has a low value when the magnetization orientations of the fixed layer and the free layer are parallel. The resistance increases when the magnetizations are antiparallel. The voltage dependence 
TABLE 1: ITRS roadmap 2013 for STT-MRAM technology requirements.

\begin{tabular}{|c|c|c|c|c|c|c|c|c|}
\hline Year of production & 2013 & 2014 & 2015 & 2016 & 2017 & 2018 & 2019 & 2020 \\
\hline Technology node $F(\mathrm{~nm})$ & 90 & 90 & 65 & 65 & 45 & 45 & 45 & 32 \\
\hline Cell size area factor a in multiples of $F 2$ & 28 & 28 & 20 & 20 & 15 & 14 & 14 & 10 \\
\hline Typical cell size $\left(\mu \mathrm{m}^{2}\right)$ & 0.23 & 0.23 & 0.08 & 0.08 & 0.030 & 0.028 & 0.028 & 0.010 \\
\hline Material technology & IMA & IMA & IMA & IMA & $P M A$ & $P M A$ & $P M A$ & PMA \\
\hline Switching current $(\mu \mathrm{A})$ & 340 & 310 & 200 & 175 & 120 & 120 & 100 & 50 \\
\hline Write energy (pJ/bit) & 4.3 & 3.9 & 2.5 & 2.5 & 1.2 & 1.2 & 1 & 0.3 \\
\hline Active area per cell $\left(\mu \mathrm{m}^{2}\right)$ & 0.021 & 0.019 & 0.01 & 0.008 & 0.006 & 0.006 & 0.005 & 0.004 \\
\hline RA product $\left(\mathrm{Ohm}-\mu \mathrm{m}^{2}\right)$ & 13.5 & 13.5 & 12.5 & 11 & 10 & 10 & 10 & 10 \\
\hline MR ratio (\%) & 120 & 120 & 120 & 120 & 150 & 150 & 150 & 150 \\
\hline Nonvolatile data retention (years) & $>10$ & $>10$ & $>10$ & $>10$ & $>10$ & $>10$ & $>10$ & $>10$ \\
\hline Write endurance (read/write cycles) & $>1 E 12$ & $>1 E 12$ & $>1 E 12$ & $>1 E 12$ & $>1 E 12$ & $>1 E 12$ & $>1 E 12$ & $>1 E 15$ \\
\hline Endurance (years at bias) & $>10$ & $>10$ & $>10$ & $>10$ & $>10$ & $>10$ & $>10$ & $>10$ \\
\hline
\end{tabular}

of MTJ resistance is different for the parallel and antiparallel states. A high resistance has a larger voltage dependence than a low resistance [38].

The voltage dependence of MTJ resistance has been established by using conductance. There are three conductance models [40-42] which are most frequently used.

Brinkman et al. [40] presented bias-dependent conductance in 1970. Brinkman et al. developed a voltage-dependent conductance model that is composed of barrier thickness and barrier height and has the following parabolic relationship:

$$
G(V)=G_{0}\left(1-\frac{A_{0} \Delta \Phi}{8 \Phi^{3 / 2}}+\frac{9 A_{0}^{2}}{128 \Phi}(e V)^{2}\right),
$$

where $G_{0}=\left(q^{2} / t h^{2}\right) \sqrt{2 q m \phi} \exp (-t \sqrt{8 m q \phi} / \hbar)$ is the conductance at zero bias, $t$ is the barrier thickness, $\phi$ is the potential, $h$ is Planck's constant, $q$ is the electron charge, $m$ is the electron mass, and $A_{0}=4 t \sqrt{2 m} / 3 \hbar$.

Julliere's model [41] describes the relative conductance using the spin polarization $P$ of conduction electrons as in (5) and considers only two states (parallel and antiparallel):

$$
\mathrm{TMR}=\frac{2 P_{1} P_{2}}{1-P_{1} P_{2}},
$$

where $P_{i}$ is the polarization of layer $i$.

Additionally, Slonczewski [42] demonstrated that the conductance is a function of the angle between the magnetizations of the two ferromagnetic layers as given in (6), whereas the voltage dependency is not considered:

$$
G(\theta)=\bar{G}\left(1+P_{1} P_{2} \cos \theta\right),
$$

where $\theta$ is the angle between the magnetization of the free layer and fixed layer.

Many of behavioral models of voltage-dependent MTJ resistance are based on these three conductance models. Several behavioral models for MTJ that describe the voltage dependence of MTJ resistance have been reported [8, 9, 4346].

Zhao et al. [8] used Brinkman's conductance model [40] for behavioral model of an MTJ. The model is developed

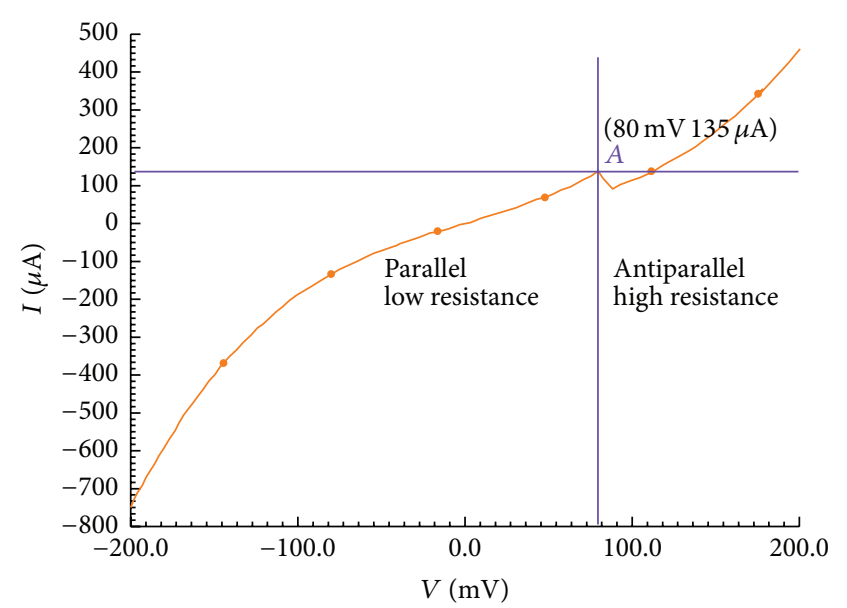

FIGURE 5: SPICE simulation results of the behavioral model [8]. Reprinted from [8].

in the Verilog-A language, and, thus, it is compatible with circuit-level simulators, such as SPICE. The DC simulation results show the parabolic relationship between the resistance and voltage, as shown in Figure 5.

Madec et al. [9] developed a tunneling current model that integrated three conductance models: Julliere's model [41], Slonczewski's model [42], and Brinkman's model [40]. In addition, the TMR fitting model shown in the following is also integrated together:

$$
\operatorname{TMR}(V)=\operatorname{TMR}_{0} \frac{1}{1+\left(V / V_{h}\right)^{2}},
$$

where $\mathrm{TMR}_{0}$ is the TMR at zero bias and $V_{h}$ is a fitting parameter corresponding to the voltage at which the TMR is halved.

Madec's model can be accurate because three conductance models are integrated into one model to complement each other, as shown in Figure 6. If the low resistance is assumed to have a constant value, as indicated by many experimental data, however, the voltage dependence of the conductance or TMR is represented only by high resistance. 


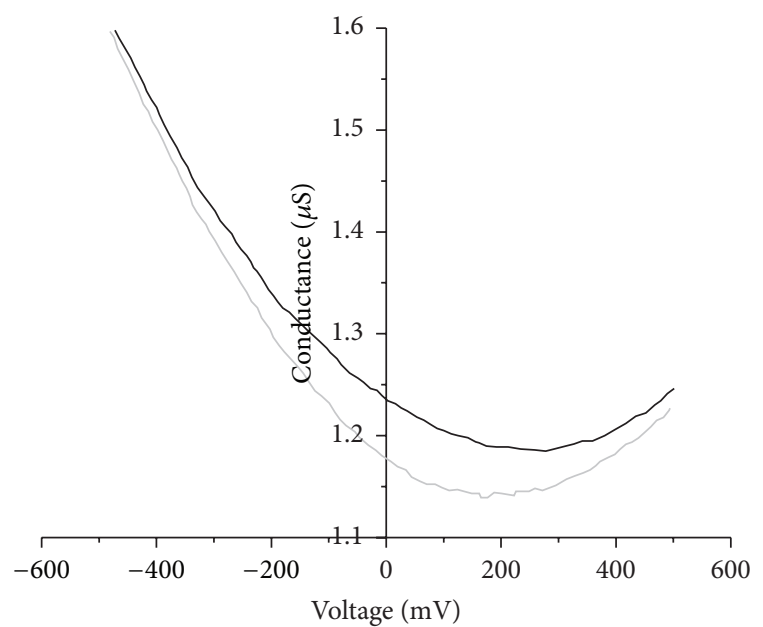

FIGURE 6: Simulated voltage-dependent conductance model of [9]. The black curve corresponds to the parallel state and the gray one corresponds to the antiparallel state. Reprinted from [9].

Therefore, Madec's approach, which uses both Brinkman's conductance model and the voltage-dependent TMR model to represent the voltage dependence of the conductance, is likely to overestimate the voltage dependency of the MTJ resistance.

Most behavioral models of voltage-dependent MTJ resistance are based on Brinkman's model. Other behavioral models use a fitting scheme to represent the voltage dependence of MTJ resistance. Many fitted behavioral models express the conductance or TMR as having a parabolic or exponential relationship with voltage $[45,46]$. These behavioral models are described by simple equations to simulate the characteristics of MTJ resistance. Thus, they are useful and efficient in running simulations on large arrays of STT-MRAM cells.

Another type of modeling method is macromodeling, which represents the characteristics using circuit elements [10-12, 15, 18, 47-49]. Panagopoulos et al. [10] implemented the Landau-Lifshitz-Gilbert (LLG) equation using linear capacitors with voltage-dependent current sources and resistors, as shown in Figure 7. Based on Kirchhoff's law, the dynamics of magnetization over time can be solved by capacitors and current sources. In this model, the out-ofplane angle $\theta$ and in-plane angle $\phi$ are calculated and used to represent the MTJ resistance as follows:

$$
\begin{aligned}
R(\theta, V, T)= & \frac{\sin (c T)}{c T}\left[P_{3} \theta^{3}+P_{2} \theta^{2}+P_{1} \theta+R_{P}\right] \\
& \cdot\left(1-\frac{\operatorname{abs}(V)}{\text { Slope }}\right) \cdot 10^{S\left(T_{\text {ox }}-T_{\text {ox } x, 0}\right)},
\end{aligned}
$$

where $V$ is the applied voltage, $T$ is the temperature, $c$ is a material dependent parameter, $R_{P}$ is the tunneling resistance in the parallel state, and $T_{\mathrm{ox}}$ is the oxide thickness.

Although the in-plane angle is not used to calculate STT and resistance, this macromodel represents the voltage dependence of the MTJ resistance well.

The voltage dependence of MTJ resistance can be implemented by a physics-based macromodel, as described above.

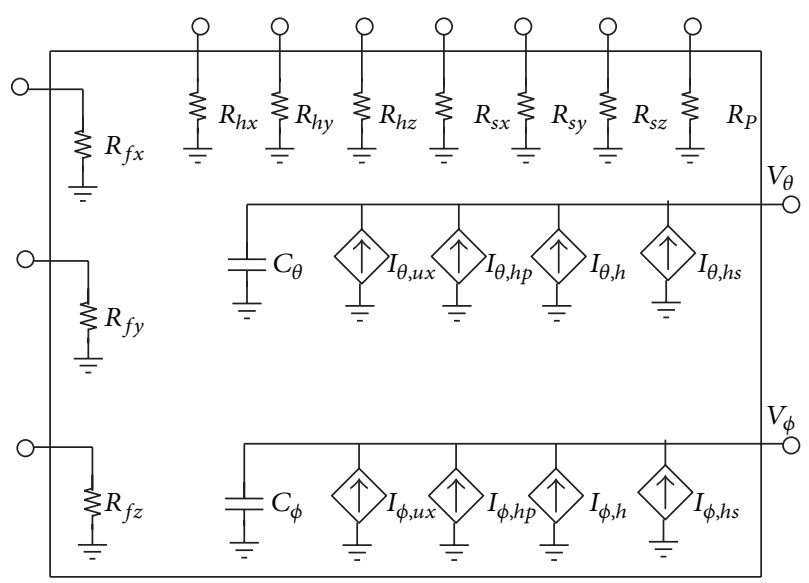

FIGURE 7: SPICE subcircuit component to implement the LLG equation. Reprinted from [10].

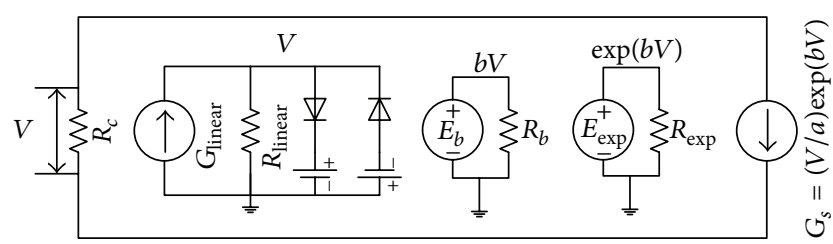

FIGURE 8: Schematic representation of the subcircuit used for representing the nonlinear voltage dependence of the MTJ. Reprinted from [11].

Another way to implement a macromodel is to represent the characteristics by fitting to experimental data. As noted above, the voltage dependency is different for the parallel and antiparallel states. The voltage dependency of high resistance is typically larger than that of low resistance, and it can be fitted to a Gaussian distribution or parabolic function of the voltage. In other words, MTJ resistance has a high value at zero bias and decreases with increasing voltage.

Mukherjee and Kurinec [11] implemented the nonlinear voltage dependency of MTJ resistance as a parallel combination of a linear resistor and voltage-controlled current source, as shown in Figure 8. The voltage-controlled current source is used to fit the exponential voltage dependence, and the macromodel describes the voltage-dependent resistance by adjusting the fitting parameters $a, b$, and $c$,

$$
\frac{1}{R_{p(a p)}}=\frac{1}{c_{p(a p)}}+\frac{1}{a_{p(a p)} \exp \left(-b_{p(a p)} V\right)} .
$$

Our group [12] also reported a macromodel of a threeterminal subcircuit that reproduces the asymmetric voltage dependency of MTJ resistance, as shown in Figure 9. In this research, low resistance is assumed to have a constant value, and high resistance is fitted to a Lorentzian fitting expression. The hysteretic characteristics are implemented by voltage-controlled voltage sources and linear resistors. The Lorentzian distribution of voltage dependency is simply composed of voltage-controlled resistors. The macromodel successfully reproduces the asymmetric voltage dependency 


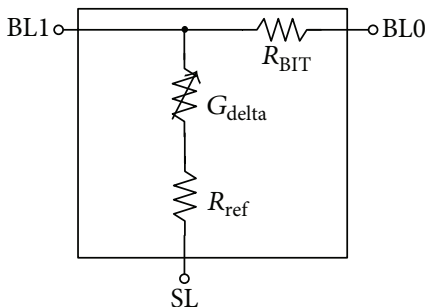

(a)

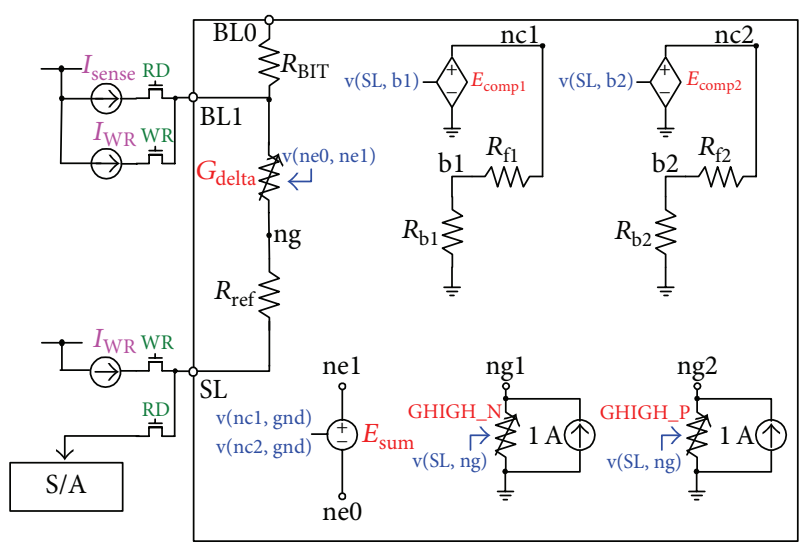

(b)

FIGURE 9: Schematics of (a) a block diagram having a three-terminal subcircuit and (b) the entire subcircuit contained in the macromodel. Reprinted from [12].

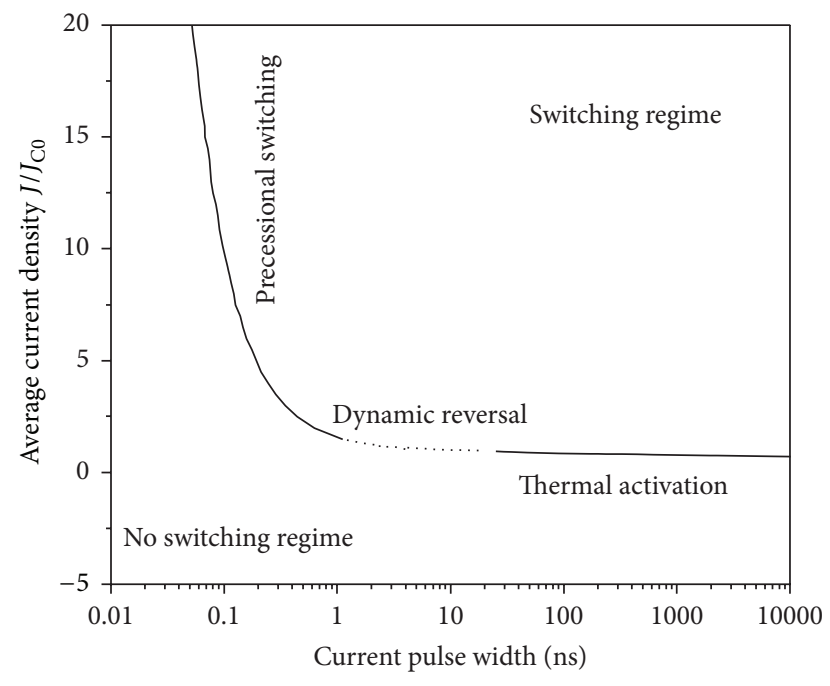

FIGURE 10: Switching phase diagram reprinted from [13].

of experimental data. The model is shown to be easily integrated with a CMOS circuit for STT-MRAM simulation.

3.2. Switching Behavior Modeling. It has been found that switching behavior of MTJs can be classified into three distinct modes as a function of current pulse width, as shown in Figure 10: thermal activation, dynamic reversal, and precessional switching [13]. The thermal activation switching mode that has generally been used for MTJs is driven in a long current pulse regime. As the demand for low-power dissipation and high-speed operation has increased, the STTbased precessional switching mode in the short current pulse regime has become more important, as has dynamic reversal switching in the intermediate current pulse regime.

The switching behavior of an MTJ can be expressed by the relationship between the bias current and switching time. The dynamic switching behaviors can be divided into two categories by the critical current, $I_{C 0}$, and are dependent on the current pulse width.

When the bias current is smaller than $I_{C 0}$ and the current pulse width is sufficiently long, Neel-Brown's model can be applied to the switching probability of MTJs [50]. In this case, the switching time, $\tau_{\text {thermal }}$, is determined by the ratio between the energy barrier and thermal fluctuation corrected by the current as

$$
\tau_{\text {thermal }}=\tau_{0} \exp \left(\frac{E}{k_{\mathrm{B}} T}\left(1-\frac{I}{I_{\mathrm{C} 0}}\right)\right), \quad\left(I<I_{\mathrm{C} 0}\right),
$$

where $\tau_{0}$ is the inverse of the attempt frequency, $E=$ $\mu_{0} M_{S} V H_{C} / 2$ is the energy barrier, $k_{\mathrm{B}}$ is the Boltzmann constant, $T$ is the temperature, $V$ is the volume of the free layer, and $H_{C}$ is the coercive field. The switching behavior is dominated by thermal activation in this region.

In the other case, when the bias current exceeds $I_{C 0}$ and the current pulse width is under a few nanoseconds, the switching behavior is nearly independent of the thermal activation and is dominated by STT. In this regime, the switching behavior turns into precessional switching, and the switching time is inversely proportional to the bias current as expressed by Sun's model [51]:

$$
\begin{aligned}
\tau_{\text {prec }}=\frac{\ln \left(\pi / 2 \theta_{0}\right)}{\alpha \gamma\left(H_{\text {ext }}+H_{A}+M_{S} / 2\right)}\left(\frac{I_{C 0}}{I-I_{C 0}}\right), & \\
& \left(I>I_{C 0}\right),
\end{aligned}
$$

where $\theta_{0}=\left(k_{\mathrm{B}} T / 2 E\right)^{1 / 2}$ is the initial angular deviation of the magnetization from the easy axis.

The above two models represent the switching behavior in the thermal activation mode and precessional switching mode well, but it is difficult to represent the switching time in the dynamic reversal mode due to the complex switching behavior near $I_{\mathrm{C} 0}$. To accurately predict the MTJ switching characteristics, a unified model is required in entire current pulse width regions that include the thermal activation mode, 


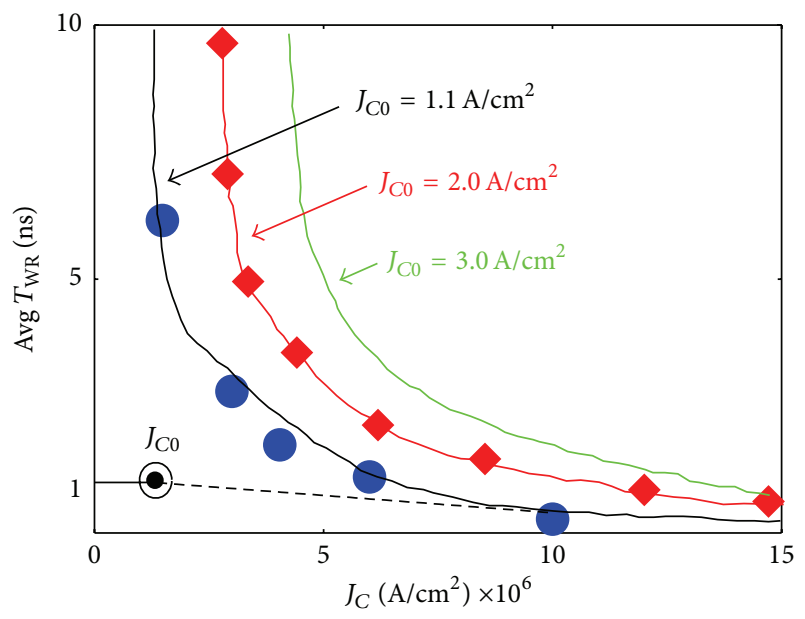

FIGURE 11: Behavioral model [14] of switching behavior for different values of $J_{C 0}$. Reprinted from [14].

precessional switching mode, and dynamic reversal mode for circuit-level simulation.

There are some behavioral models that cover the entire current pulse width region [14-16, 52, 53]. Raychowdhury et al. [14] defined the dynamic reversal region at 3-10 ns and represented it using both the thermal activation switching mode and precessional switching mode. In this model, the dynamic reversal mode is a combination of the above two switching modes and has an exponential relationship. Additionally, fitting parameters $A$ and $T_{\mathrm{PIV}}$ are introduced to allow seamless transition from one region to another as in (12c):

$$
\begin{aligned}
& J_{\mathrm{C}}^{\mathrm{THERM}}\left(T_{\mathrm{WR}}\right)=J_{\mathrm{C} 0}\left(1-\frac{1}{\Delta} \ln \left(\frac{T_{\mathrm{WR}}}{\tau_{0}}\right)\right): \\
& T_{\mathrm{WR}}>10 \mathrm{~ns}, \\
& J_{\mathrm{C}}^{\mathrm{PREC}}\left(T_{\mathrm{WR}}\right)=J_{\mathrm{C} 0}+\frac{C \ln (\pi / 2 \theta)}{T_{\mathrm{WR}}}: \quad T_{\mathrm{WR}}<3 \mathrm{~ns}, \\
& J_{\mathrm{C}}^{\text {DYN }}\left(T_{\mathrm{WR}}\right) \\
& =\frac{J_{\mathrm{C}}^{\mathrm{THERM}}\left(T_{\mathrm{WR}}\right)+J_{\mathrm{C}}^{\mathrm{PREC}}\left(T_{\mathrm{WR}}\right) \exp \left(-A\left(T_{\mathrm{WR}}-T_{\mathrm{PIV}}\right)\right)}{1+\exp \left(-A\left(T_{\mathrm{WR}}-T_{\mathrm{PIV}}\right)\right)} \\
& 3 \mathrm{~ns}<T_{\mathrm{WR}}<10 \mathrm{~ns} .
\end{aligned}
$$

This model fits the experimental data well, as shown in Figure 11. However, the slope of the switching current changes drastically according to the fitting parameter $A$.

Harms et al. [15] also presented a behavioral model of switching characteristics for the entire current pulse width region. This model is a modified form of the thermal activation switching model with a fitting parameter $t_{t-p}$ that determines when the transition from the thermal activation to precessional switching mode will occur, as described in the following:

$$
t_{p}=t_{0} \exp \left(\left(\frac{E}{k_{\mathrm{B}} T}\right)\left(1-\frac{I_{\mathrm{MTJ}}}{I_{\mathrm{C} 0}}\right)\right)+t_{t \rightarrow p}\left(\frac{I_{t \rightarrow p}}{I_{\mathrm{MTJ}}}\right)^{2} .
$$

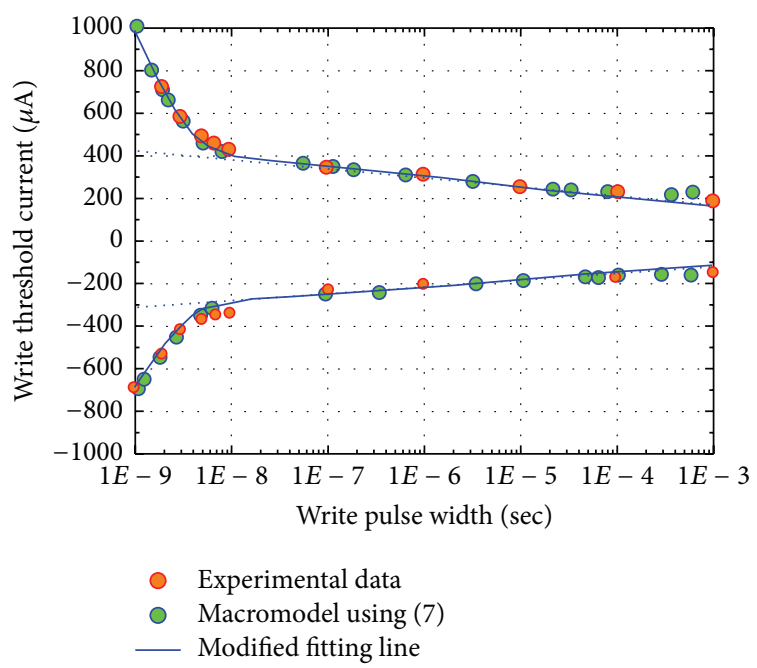

FIGURE 12: Simulation results comparing the model with experimental data. Reprinted from [15].

This model corresponds well with experimental data, as shown in Figure 12. However, it appears to omit the dynamic reversal mode and represents the precessional switching mode without physical meaning.

Recently, our group $[16,52]$ presented an analytical behavioral model that encompasses all of the switching modes at one time. A new threshold current $I_{C 1}$ is introduced, and the switching time is newly constructed so that the model can represent the characteristics of the dynamic reversal regime and the precessional switching regime according to the bias current. The model is given as [16]

$$
\tau= \begin{cases}\tau_{0} \exp \left(\frac{E}{k_{\mathrm{B}} T}\left(1-\frac{I}{I_{\mathrm{C} 0}}\right)\right), & \left(I<I_{\mathrm{C} 1}\right), \\ \delta\left[\frac{I-I_{\mathrm{C} 1}}{I_{\mathrm{C} 0}}+\frac{k_{\mathrm{B}} T}{E}\right]^{-1}, & \left(I>I_{C 1}\right),\end{cases}
$$

where $I_{C 1}=I_{C 0}\left[1-\left(k_{\mathrm{B}} T / E\right) \ln \left(\left(\delta / \tau_{0}\right)\left(E / k_{\mathrm{B}} T\right)\right)\right]$ and $\delta=$ $\ln \left(\pi / 2 \theta_{0}\right) / \alpha \gamma\left(H_{\text {ext }} \pm H_{A} \pm M_{S} / 2\right)$.

This model solved the problem of the discrepancy between the thermal activation mode and precessional switching mode. If the current is smaller than $I_{C 1}$, the model is exactly the same as Neel-Brown's model for the thermally activated switching regime. If the current starts to exceed $I_{C l}$, the model has the characteristics of both the thermal activation regime and precessional switching regime, which correspond to the dynamic reversal regime. Additionally, if the current is sufficiently large, the model converges to Sun's model for the precessional switching regime. Furthermore, this model contains no fitting parameter and represents the experimental data in the cases of parallel/antiparallel states, simultaneously, as shown in Figure 13.

In particular, we implemented another model based on the LLG equation [4] and compared its simulation time with that of our analytic model. As mentioned earlier, [3] reported that the behavioral approach is slower than the LLG approach because the conditional loops lead to a discontinuity, such that the simulator requires more time to find a solution. 


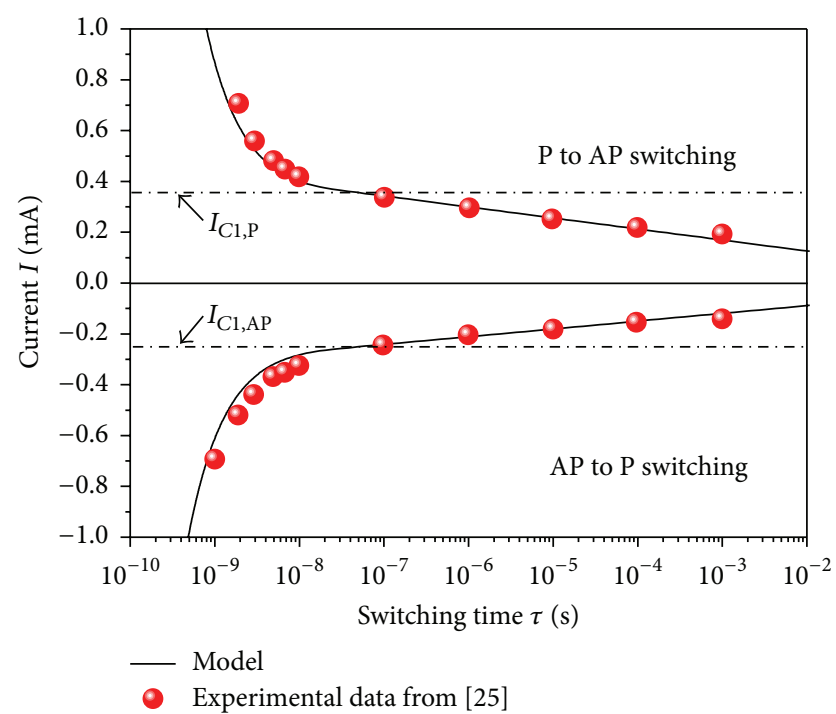

FIGURE 13: Switching current as a function of the switching time in cases of P/AP configurations. Reprinted from [16].

However, our behavioral model reached a solution 4 times faster than the LLG based model [4]. This result is due to the fact that our behavioral model consists of a unified equation (14).

In addition to the aforementioned behavioral model of switching behavior, switching characteristics can also be implemented by macromodels $[15,17,18,47,48,53]$. Shang et al. [53] suggested a new modified nodal analysis formulation to consider internal state variables for the time-dependent dynamics of MTJs. Based on Kirchhoff's law, the angles of the magnetization are chosen as internal state variables and used to define new state variable vectors or matrices. This model simplifies the complexity of existing models by introducing internal state variables. In addition, the dynamic responses of MTJ switching characteristics are accurately represented and show good agreement with the experimental data.

$\mathrm{Xu}$ et al. [17] presented a macromodel for SPICE simulation that is composed of simple RC circuits. In this model, the LLG equation is mapped onto an RC network and each resistor has functionality according to the time. The dynamics of magnetization as a function of time are divided into 4 regions, as shown in Figure 14, and some approximations are applied to each region. This model also represents the experimental data well but may exhibit discrepancies at the boundaries of different regions.

Our group [18] developed a SPICE macromodel that realizes the pulse width dependent switching characteristics. This model is noteworthy in that it implements the timedependent functionality by circuit elements despite focusing on the thermal activation mode. An imaginary RC circuit is introduced into the macromodel to measure the duration of the current pulse width, as shown in Figure 15. The macromodel determines whether to switch the state by monitoring the charging current of the $\mathrm{RC}$ circuit and comparing the output voltage of RC circuit with the critical current.

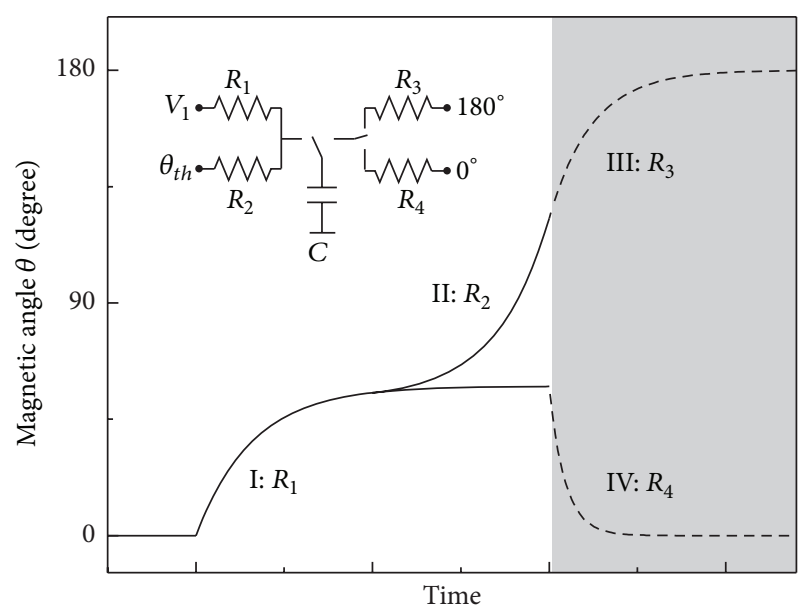

FIGURE 14: The regional RC network for dynamic SPICE simulation. Reprinted from [17].

\section{Conclusion}

In this paper, the development history of MRAM technology and various models of STT-MTJ characteristics are reviewed.

The switching technology has evolved from FIMS technology to STT switching technology. FIMS has disadvantages in terms of scalability, high power consumption, and write selection error, whereas the STT switching technique that switches the magnetization by injecting current directly into MTJ has powerful characteristics, such as high density, lowpower consumption, and low error rate.

Due to the demand for low-power dissipation and high speed, PMA devices have gained considerable attention because they are expected to provide high scalability, high thermal stability, low current density, high speed, and lowpower consumption. PMA can be obtained by adding a perpendicular polarizer to an IMA device or by using a fully perpendicular ferromagnetic layer.

The two most important characteristics of MTJ are its voltage-dependent MTJ resistance and switching behavior. Models of these characteristics can be categorized as macromodels or behavioral models. To represent the voltage dependency of MTJ resistance, models are based on the physics of STT, such as the LLG equation, or the voltage dependency of MTJ resistance is represented by fitting to experimental data.

The model must cover the entire current pulse width region for switching behavior. Some behavioral models proposed a dynamic switching behavior model by introducing a fitting parameter between the thermal activation and precessional switching regimes. Other behavioral models suggested a new threshold current to enable a seamless transition from the thermal activation regime to the precessional switching regime. Furthermore, this model has no fitting parameter and simultaneously represents the experimental data in the case of parallel/antiparallel states. There are also macromodels that describe the switching behavior of MTJs with circuit elements.

STT-MRAM is one of the most promising nonvolatile memory forms that may replace traditional memory forms, 


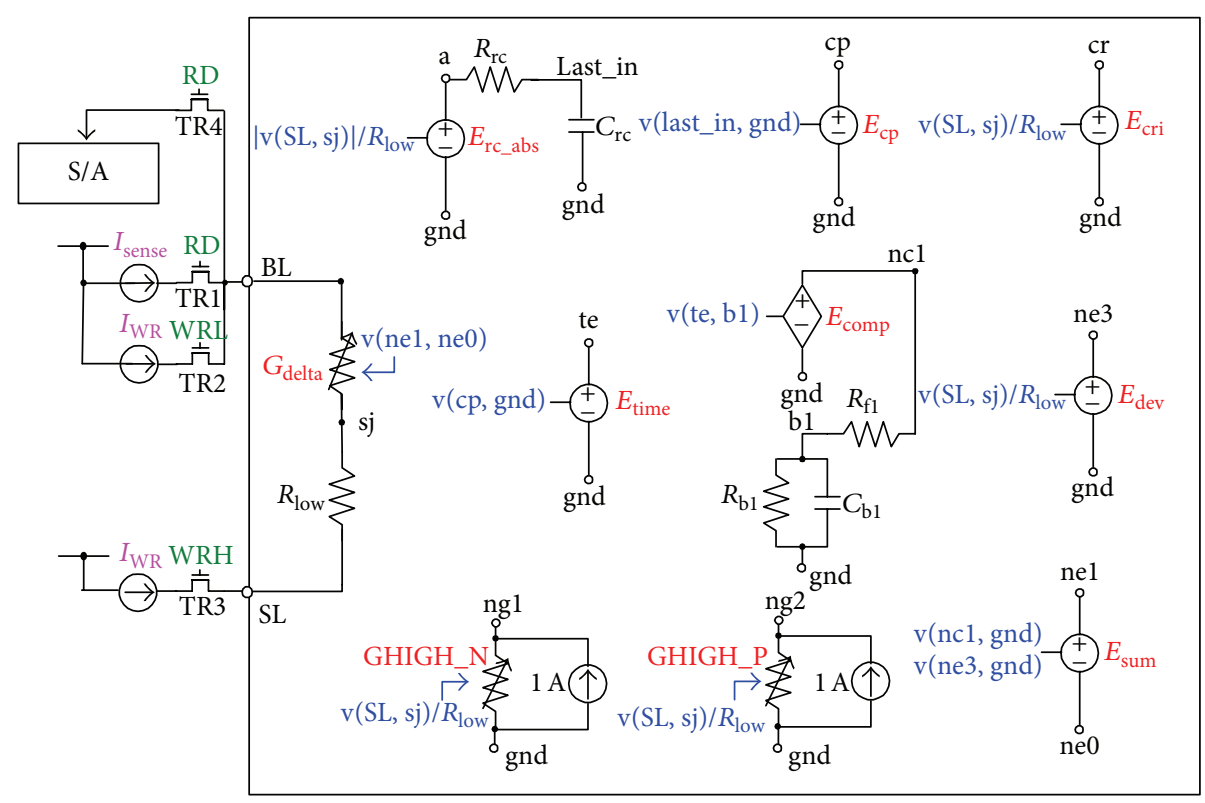

Figure 15: Schematic of the macromodel [18]. Reprinted from [18].

such as DRAM or flash memory. Advanced material technology and accurate circuit-level models of MTJ can accelerate the commercialization of STT-MRAM.

\section{Competing Interests}

The authors declare that they have no competing interests.

\section{Acknowledgments}

This research was supported by the National Research Foundation of Korea (NRF) funded by the Ministry of Science, ICT and Future Planning (no. 2014R1A2A2A01002219).

\section{References}

[1] S. Ikeda, J. Hayakawa, Y. M. Lee et al., "Magnetic tunnel junctions for spintronic memories and beyond," IEEE Transactions on Electron Devices, vol. 54, no. 5, pp. 991-1002, 2007.

[2] Y. Huai, D. Apalkov, Z. Diao et al., "Structure, materials and shape optimization of magnetic tunnel junction devices: spintransfer switching current reduction for future magnetoresistive random access memory application," Japanese Journal of Applied Physics, vol. 45, no. 5, pp. 3835-3841, 2006.

[3] K. Jabeur, F. Bernard-Granger, G. Di Pendina, G. Prenat, and B. Dieny, "Comparison of Verilog-A compact modelling strategies for spintronic devices," Electronics Letters, vol. 50, no. 19, pp. 1353-1355, 2014.

[4] A. Vatankhahghadim, S. Huda, and A. Sheikholeslami, "A survey on circuit modeling of spin-transfer-torque magnetic tunnel junctions," IEEE Transactions on Circuits and Systems I: Regular Papers, vol. 61, no. 9, pp. 2634-2643, 2014.

[5] G. Prenat, M. El Baraji, W. Guo et al., "CMOS/magnetic hybrid architectures," in Proceedings of the 14th IEEE International Conference on Electronics, Circuits and Systems (ICECS '07), pp. 190-193, Marrakech, Morocco, December 2007.
[6] G. E. Rowlands, T. Rahman, J. A. Katine et al., "Deep subnanosecond spin torque switching in magnetic tunnel junctions with combined in-plane and perpendicular polarizers," Applied Physics Letters, vol. 98, no. 10, Article ID 102509, 2011.

[7] D. C. Worledge, G. Hu, D. W. Abraham et al., "Spin torque switching of perpendicular TaCoFeBMgO-based magnetic tunnel junctions," Applied Physics Letters, vol. 98, Article ID 022501, 2011.

[8] W. Zhao, E. Belhaire, Q. Mistral et al., "Macro-model of spintransfer torque based magnetic tunnel junction device for hybrid magnetic-CMOS design," in Proceedings of the IEEE International Behavioral Modeling and Simulation Workshop (BMAS '06), pp. 40-43, San Jose, Calif, USA, September 2006.

[9] M. Madec, J.-B. Kammerer, and L. Hébrard, "Compact modeling of a magnetic tunnel junction-part II: tunneling current model," IEEE Transactions on Electron Devices, vol. 57, no. 6, pp. 1416-1424, 2010.

[10] G. D. Panagopoulos, C. Augustine, and K. Roy, "Physicsbased SPICE-compatible compact model for simulating hybrid MTJ/CMOS circuits," IEEE Transactions on Electron Devices, vol. 60, no. 9, pp. 2808-2814, 2013.

[11] S. S. Mukherjee and S. K. Kurinec, "A stable SPICE macromodel for magnetic tunnel junctions for applications in memory and logic circuits," IEEE Transactions on Magnetics, vol. 45, no. 9, pp. 3260-3268, 2009.

[12] S. Lee, H. Lee, S. Kim, S. Lee, and H. Shin, "A novel macro-model for spin-transfer-torque based magnetic-tunneljunction elements," Solid-State Electronics, vol. 54, no. 4, pp. 497-503, 2010.

[13] Z. Diao, Z. Li, S. Wang et al., "Spin-transfer torque switching in magnetic tunnel junctions and spin-transfer torque random access memory," Journal of Physics Condensed Matter, vol. 19, no. 16, Article ID 165209, 2007.

[14] A. Raychowdhury, D. Somasekhar, T. Karnik, and V. De, "Design space and scalability exploration of 1T-1STT MTJ memory arrays in the presence of variability and disturbances," in Proceedings of the IEEE International Electron Devices 
Meeting. Technical Digest (IEDM '09), Baltimore, Md, USA, December 2009.

[15] J. D. Harms, F. Ebrahimi, X. Yao, and J.-P. Wang, "SPICE macromodel of spin-torque-transfer-operated magnetic tunnel junctions," IEEE Transactions on Electron Devices, vol. 57, no. 6, pp. 1425-1430, 2010.

[16] H. Lim, S. Lee, and H. Shin, "Unified analytical model for switching behavior of magnetic tunnel junction," IEEE Electron Device Letters, vol. 35, no. 2, pp. 193-195, 2014.

[17] Z. Xu, C. Yang, M. Mao, K. B. Sutaria, C. Chakrabarti, and Y. Cao, "Compact modeling of STT-MTJ devices," Solid-State Electronics, vol. 102, pp. 76-81, 2014.

[18] S. Kim, S. Lee, and H. Shin, "Advanced macro-model with pulse-width dependent switching characteristic for spin transfer torque based magnetic-tunnel-junction elements," Japanese Journal of Applied Physics, vol. 49, no. 4, Article ID 04DM07, 2010.

[19] M. Durlam, D. Addie, J. Akerman et al., "A $0.18 \mu \mathrm{m} 4 \mathrm{Mb}$ toggling MRAM," IEDM Technical Digest, IEEE Press, New York, NY, USA, 2003.

[20] I. L. Prejbeanu, M. Kerekes, R. C. Sousa et al., "Thermally assisted MRAM," Journal of Physics Condensed Matter, vol. 19, no. 16, Article ID 165218, 2007.

[21] J. Z. Sun, "Spin-current interaction with a monodomain magnetic body: a model study," Physical Review B, vol. 62, article 570, 2000.

[22] H. Sato, M. Yamanouchi, K. Miura et al., "Junction size effect on switching current and thermal stability in $\mathrm{CoFeB} / \mathrm{MgO}$ perpendicular magnetic tunnel junctions," Applied Physics Letters, vol. 99, no. 4, Article ID 042501, 2011.

[23] J. H. Park, Y. Kim, W. C. Lim et al., "Enhancement of data retention and write current scaling for sub-20 $\mathrm{nm}$ STT-MRAM by utilizing dual interfaces for perpendicular magnetic anisotropy," in Proceedings of the Symposium on VLSI Technology (VLSIT '12), pp. 57-58, Honolulu, Hawaii, USA, July 2012.

[24] T. Kishi, H. Yoda, T. Kai et al., "Lower-current and fast switching of A perpendicular TMR for high speed and high density spin-transfer-torque MRAM," in Proceedings of the IEEE International Electron Devices Meeting (IEDM '08), pp. 1-4, San Francisco, Calif, USA, December 2008.

[25] P. K. Amiri, Z. M. Zeng, J. Langer et al., "Switching current reduction using perpendicular anisotropy in $\mathrm{CoFeB}-\mathrm{MgO}$ magnetic tunnel junctions," Applied Physics Letters, vol. 98, no. 11, Article ID 112507, 2011.

[26] H. Zhao, B. Glass, P. K. Amiri et al., "Sub-200 ps spin transfer torque switching in in-plane magnetic tunnel junctions with interface perpendicular anisotropy," Journal of Physics D: Applied Physics, vol. 45, no. 2, Article ID 025001, 2012.

[27] N. Nishimura, T. Hirai, A. Koganei et al., "Magnetic tunnel junction device with perpendicular magnetization films for high-density magnetic random access memory," Journal of Applied Physics, vol. 91, no. 8, pp. 5246-5249, 2002.

[28] H. Ohmori, T. Hatori, and S. Nakagawa, "Perpendicular magnetic tunnel junction with tunneling magnetoresistance ratio of $64 \%$ using $\mathrm{MgO}$ (100) barrier layer prepared at room temperature," Journal of Applied Physics, vol. 103, no. 7, Article ID 07A911, 2008.

[29] H. Liu, D. Bedau, D. Backes, J. A. Katine, J. Langer, and A. D. Kent, "Ultrafast switching in magnetic tunnel junction based orthogonal spin transfer devices," Applied Physics Letters, vol. 97, no. 24, Article ID 242510, 2010.
[30] S. Ikeda, K. Miura, H. Yamamoto et al., "A perpendicularanisotropy $\mathrm{CoFeB}-\mathrm{MgO}$ magnetic tunnel junction," Nature Materials, vol. 9, no. 9, pp. 721-724, 2010.

[31] J. C. Slonczewski, "Current-driven excitation of magnetic multilayers," Journal of Magnetism and Magnetic Materials, vol. 159, no. 1-2, pp. L1-L7, 1996.

[32] J. C. Slonczewski, "Currents, torques, and polarization factors in magnetic tunnel junctions," Physical Review B, vol. 71, no. 2, Article ID 024411, 2005.

[33] L. Liu, T. Moriyama, D. C. Ralph, and R. A. Buhrman, "Reduction of the spin-torque critical current by partially canceling the free layer demagnetization field," Applied Physics Letters, vol. 94, Article ID 122508, 2009.

[34] K. L. Wang, J. G. Alzate, and P. Khalili Amiri, "Low-power nonvolatile spintronic memory: STT-RAM and beyond," Journal of Physics D: Applied Physics, vol. 46, no. 8, Article ID 074003, 2013.

[35] M. T. Rahman, A. Lyle, P. Khalili Amiri et al., "Reduction of switching current density in perpendicular magnetic tunnel junctions by tuning the anisotropy of the CoFeB free layer," Journal of Applied Physics, vol. 111, no. 7, Article ID 07C907, 2012.

[36] J. Z. Sun, R. P. Robertazzi, J. Nowak et al., "Spin-torque switchable perpendicular magnetic junctions for solid-state memory," in Proceedings of the 69th Annual Device Research Conference (DRC '11), pp. 171-174, Santa Barbara, Calif, USA, June 2011.

[37] ITRS Roadmap, 2013, http://www.itrs2.net/2013-itrs.html.

[38] S. Zhang, P. M. Levy, A. C. Marley, and S. S. P. Parkin, "Quenching of magnetoresistance by hot electrons in magnetic tunnel junctions," Physical Review Letters, vol. 79, no. 19, pp. 3744-3747, 1997.

[39] S. Yuasa, T. Nagahama, A. Fukushima, Y. Suzuki, and K. Ando, "Giant room-temperature magnetoresistance in single-crystal $\mathrm{Fe} / \mathrm{MgO} / \mathrm{Fe}$ magnetic tunnel junctions," Nature Materials, vol. 3, no. 12, pp. 868-871, 2004.

[40] W. F. Brinkman, R. C. Dynes, and J. M. Rowell, “Tunneling conductance of asymmetrical barriers," Journal of Applied Physics, vol. 41, article 1915, 1970.

[41] M. Julliere, "Tunneling between ferromagnetic films," Physics Letters A, vol. 54, no. 3, pp. 225-226, 1975.

[42] J. C. Slonczewski, "Conductance and exchange coupling of two ferromagnets separated by a tunneling barrier," Physical Review $B$, vol. 39, no. 10, pp. 6995-7002, 1989.

[43] W. Guo, G. Prenat, V. Javerliac et al., "SPICE modelling of magnetic tunnel junctions written by spin-transfer torque," Journal of Physics D, vol. 43, no. 21, Article ID 215001, 2010.

[44] S. Verma, S. Kaundal, and B. K. Kaushik, "Modeling of in-plane magnetic tunnel junction for mixed mode simulations," IEEE Transactions on Magnetics, vol. 50, no. 8, Article ID 4400307, 7 pages, 2014.

[45] K. Ono, T. Kawahara, R. Takemura et al., "A disturbance-free read scheme and a compact stochastic-spin-dynamics-based MTJ circuit model for Gb-scale SPRAM," in Proceedings of the IEEE International Electron Devices Meeting (IEDM '09), pp. 14, Baltimore, Md, USA, December 2009.

[46] R. Garg, D. Kumar, N. Jindal, N. Negi, and C. Ahuja, "Behavioural model of spin torque transfer magnetic tunnel junction, using Verilog-A," International Journal of Advanced Scientific and Technical Research, vol. 1, no. 6, 2012.

[47] Y. Chen, X. Wang, H. Li, H. Xi, Y. Yan, and W. Zhu, "Design margin exploration of spin-transfer torque RAM (STT-RAM) in scaled technologies," IEEE Transactions on Very Large Scale Integration (VLSI) Systems, vol. 18, no. 12, pp. 1724-1734, 2010. 
[48] N. Sakimura, R. Nebashi, Y. Tsuji et al., "High-speed simulator including accurate MTJ models for spintronics integrated circuit design," in Proceedings of the IEEE International Symposium on Circuits and Systems, pp. 1971-1974, IEEE, Seoul, South Korea, May 2012.

[49] S. Manipatruni, D. E. Nikonov, and I. A. Young, "Vector spin modeling for magnetic tunnel junctions with voltage dependent effects," Journal of Applied Physics, vol. 115, no. 17, Article ID 17B754, 2014.

[50] Z. Li and S. Zhang, "Thermally assisted magnetization reversal in the presence of a spin-transfer torque," Physical Review B, vol. 69, Article ID 134416, 2004.

[51] R. H. Koch, J. A. Katine, and J. Z. Sun, “Time-resolved reversal of spin-transfer switching in a nanomagnet," Physical Review Letters, vol. 92, no. 8, Article ID 088302, 2004.

[52] H. Lim, S. Lee, and H. Shin, "Advanced circuit-level model for temperature-sensitive read/write operation of a magnetic tunnel junction," IEEE Transactions on Electron Devices, vol. 62, no. 2, pp. 666-672, 2015.

[53] Y. Shang, W. Fei, and H. Yu, "Analysis and modeling of internal state variables for dynamic effects of nonvolatile memory devices," IEEE Transactions on Circuits and Systems. I. Regular Papers, vol. 59, no. 9, pp. 1906-1918, 2012. 


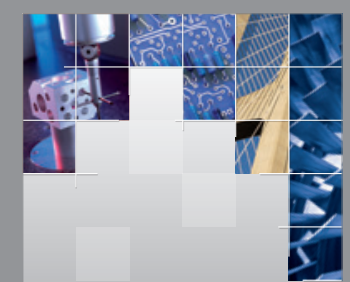

\section{Enfincering}
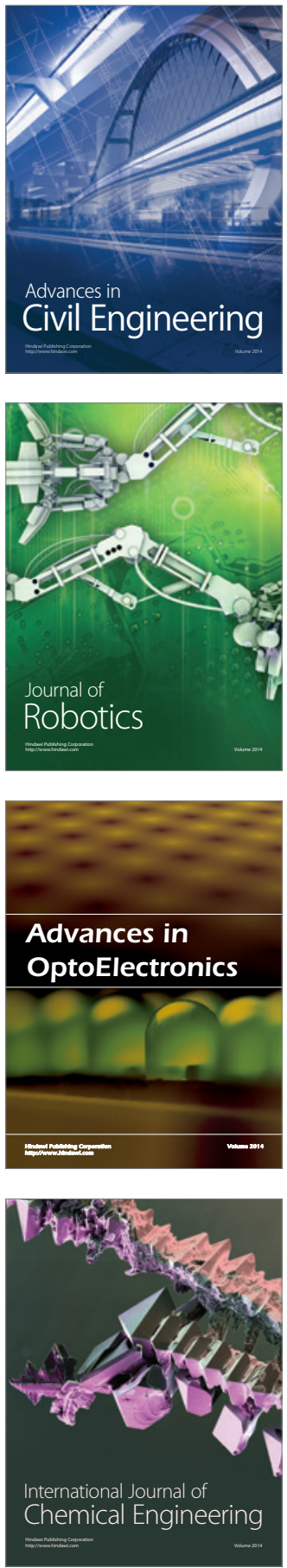

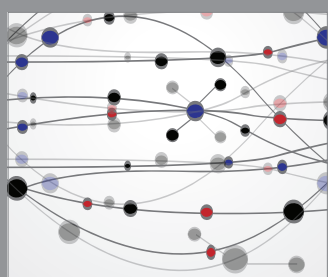

The Scientific World Journal

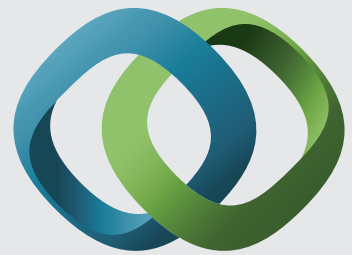

\section{Hindawi}

Submit your manuscripts at

http://www.hindawi.com
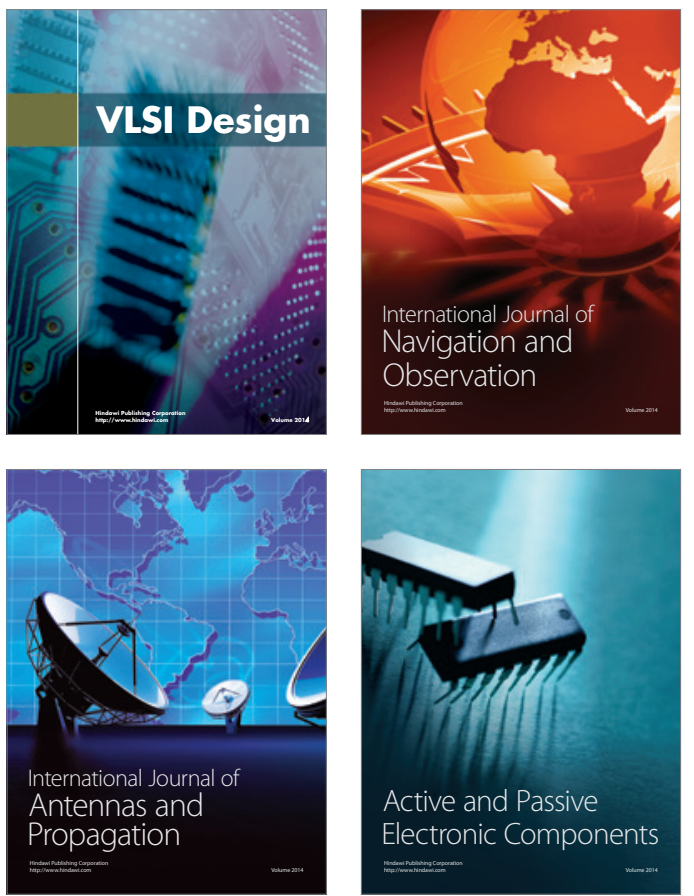
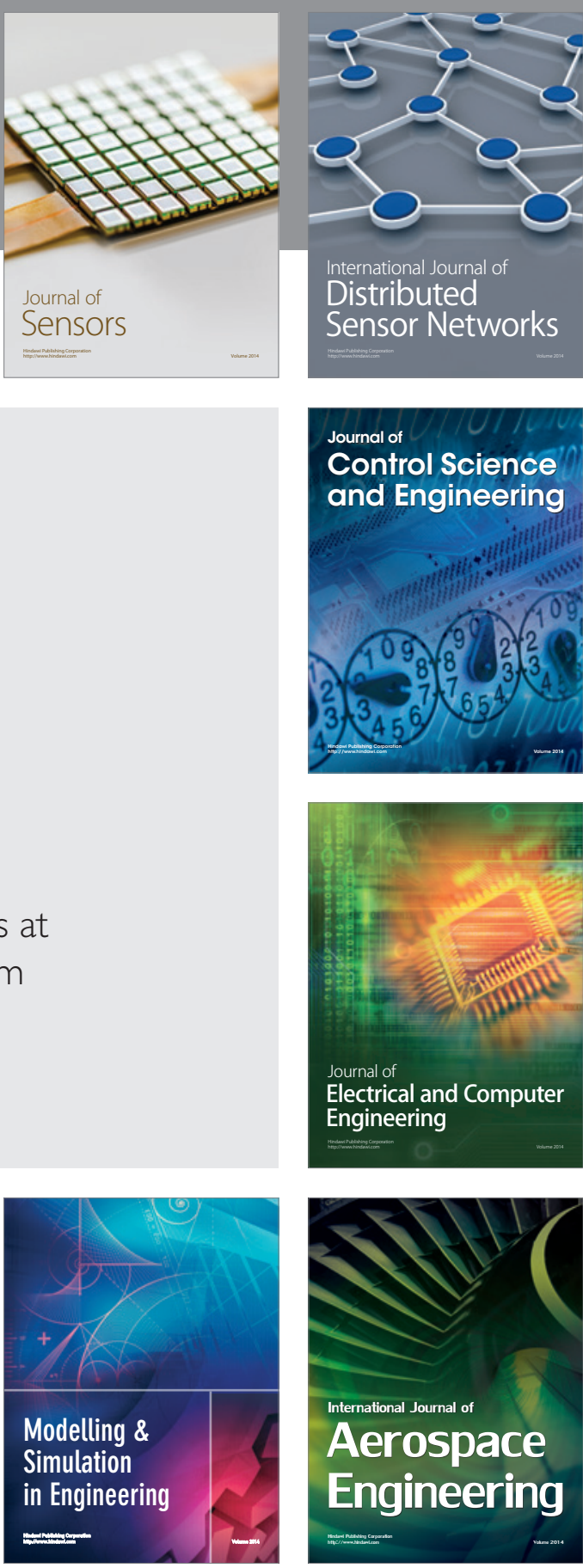

International Journal of

Distributed

Sensor Networks

Journal of

Control Science

and Engineering
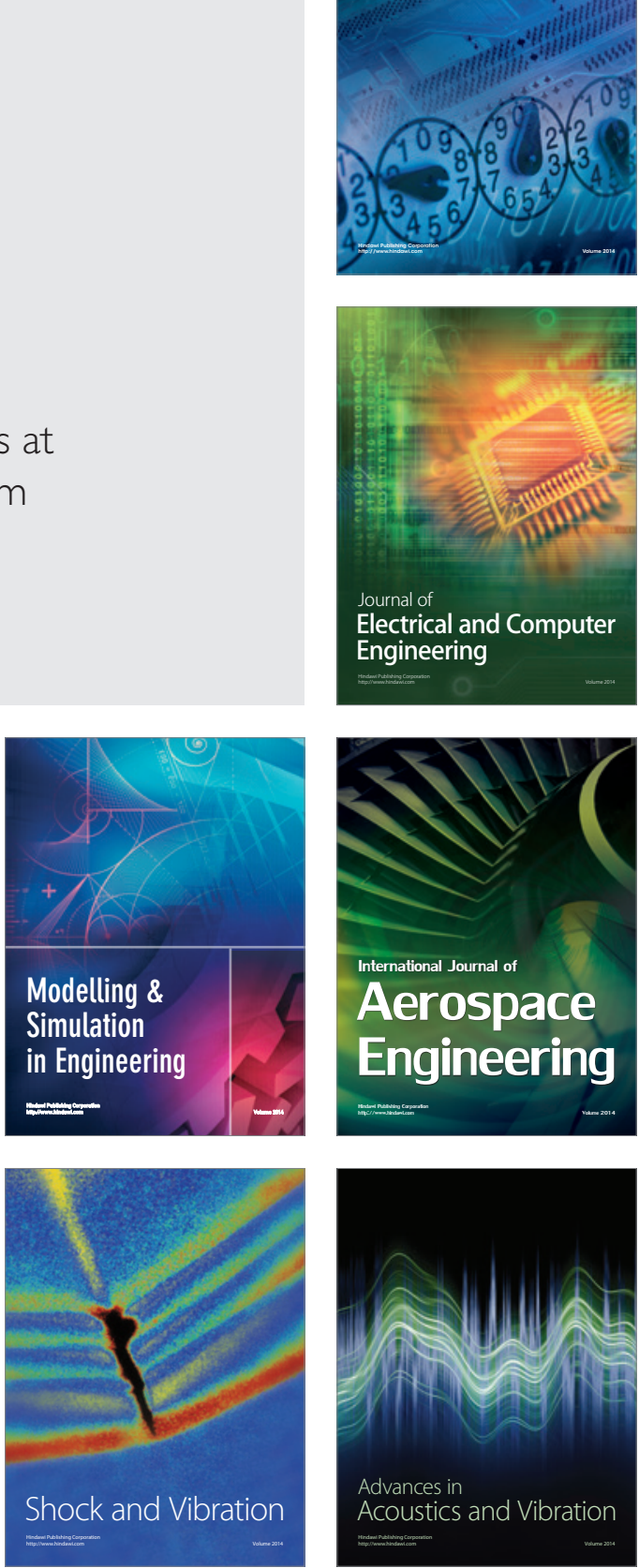